\title{
LAGRANGIAN SYMMETRIES OF CHERN-SIMONS THEORIES
}

\author{
Andrzej BOROWIEC* \\ Marco FERRARIS \\ Mauro FRANCAVIGLIA \\ Dipartimento di Matematica \\ Università di Torino
}

Via C. Alberto 10, 10123 TORINO (ITALY)

July 15, 1998

\begin{abstract}
This paper analyses the Noether symmetries and the corresponding conservation laws for Chern-Simons Lagrangians in dimension $d=3$. In particular, we find an expression for the superpotential of ChernSimons gravity. As a by-product the general discussion of superpotentials for 3rd order natural and quasi-natural theories is also given.

PACS number(s): 04.20.Fy, 11.10Kk, 11.30.-j, 04.20.Cv
\end{abstract}

\section{Introduction}

The interplay between symmetries, conservation laws and variational principles for Lagrangian dynamical systems have been under consideration for a long time, starting from the celebrated work by E. Noether. Our aim in this paper is to calculate conserved quantities for Chern-Simons gravity in dimension $d=3$. Chern-Simons theories exhibit in fact many interesting and important properties. First of all, they are based on secondary characteristic classes discovered in [1] and there is some hope that they will give new topological invariants for knots and three-manifolds. Moreover, they

\footnotetext{
* On leave from the Institute of Theoretical Physics, University of Wrocław, pl. Maksa Borna 9, PL-50-204 WROCŁAW, POLAND (e-mail: borow@ift.uni.wroc.pl).
} 
appeared in physics as natural mass terms for gauge theories and for gravity in dimension three, and after quantization they lead to a quantized coupling constant as well as a mass [2]. They have also found applications to a large variety of physical problems [3], among which we quote anyons and quantum Hall effect; when the gauge group is the inhomogeneous Lorentz group then such a theory is equivalent to standard gravity. Chern-Simons gauge theory is also an example of a topological field theory [ [- It should also be remarked that, as it has been recently shown, Chern-Simons Lagrangians are the only obstructions to an equivariant inverse problem of the calculus of variations in $d=3$ [5]. Finally, the Chern-Simons term is related to the anomaly cancellation problem in $d=2$ conformal field theories [6].

Our paper is organized as follows. For our later convenience, we shall start from a short, elementary and self-contained exposition of a variational approach to the problem of conservation laws in field theories. A more systematic approach can be found e.g. in [17, 8, 9, 10, 11], while for a rigorous mathematical presentation 1 we refer the reader to [12, 13, 14, 15]. In section 3 we collect some material from our earlier papers concerning superpotentials and in particular we shall list the explicit formulae which are necessary to calculate them in all theories of order at most three. Section 4 deals with Chern-Simons gauge Lagrangians and we discuss how general formulae for superpotentials apply in this case. Some results of section 4 are finally used in section 5 to calculate the energy-momentum complex for Chern-Simons gravity.

\section{Preliminaries}

Let us consider a field theory over a spacetime manifold $M$ of arbitrary dimension $d$, describing the behaviour of an arbitrary field $\phi$ by means of a Lagrangian $L \equiv L\left(\phi, \partial_{x} \phi, \ldots\right)$ of arbitrary order. The action functional will be

$$
I(\phi)=\int_{D} L d x
$$

where the integration is performed over a domain $D$ of the manifold $M$ of independent variables $x \equiv\left(x^{\mu}\right)$; the field configuration (i.e., the set of all dependent variables) is represented here by $\phi$. P Variation (i.e. a functional derivative) $\delta I$ of $I$ with respect to an arbitrary (infinitesimal) variation $\delta \phi$

\footnotetext{
${ }^{1}$ It involves a variational calculus on jet bundles.

${ }^{2}$ For simplicity we drop an internal field index, e.g. $\phi^{A}$
} 
of $\phi$ leads to the variation $\delta L$ of the Lagrangian itself. This, in turn, can be rearranged into two parts according to the "first variation formula" [

$$
\delta L=\frac{\delta L}{\delta \phi} \delta \phi+\partial_{\mu} \varrho^{\mu}
$$

The first term consists of the Euler-Lagrange expression (field equations):

$$
\frac{\delta L}{\delta \phi}=\frac{\partial L}{\partial \phi}-\partial_{\mu}\left(\frac{\partial L}{\partial \phi_{\mu}}\right)+\partial_{\mu} \partial \nu\left(\frac{\partial L}{\partial \phi_{\mu \nu}}\right)-\ldots
$$

and therefore vanishes on shell, i.e. when the field $\phi$ satisfies the equations of motion. The second part (boundary term) is a divergence of $\varrho^{\mu} \equiv \varrho^{\mu}(\phi, \delta \phi)$, where

$$
\varrho^{\mu}=\left[\frac{\partial L}{\partial \phi_{\mu}}-\partial_{\nu}\left(\frac{\partial L}{\partial \phi_{\mu \nu}}\right)\right] \delta \phi+\frac{\partial L}{\partial \phi_{\mu \nu}} \delta \phi_{\nu}+\ldots
$$

This boundary term is usally neglected since it does not contribute to the equations of motion (and it is killed on the boundary $\partial D$ after imposing suitable boundary conditions on $\delta \phi$ ). However, this second contribution is physically important: in fact, it does contribute to conservation laws.

Recall that a variation, say $\delta_{*} \phi$, is called an (infinitesimal) symmetry of the action $I$ if the corresponding variation $\delta_{*} L$ can be written as a divergence (i.e., $\delta_{*} L=\partial_{\mu} \tau^{\mu}$ ) without using the equations of motion. Of course, this depends heavily on the transformation properties of $L$ : for example, if the Lagrangian transforms as a scalar (i.e. $L(\phi)=L\left(\phi+\delta_{*} \phi\right)$ ), then one has $\delta_{*} L=0$.

Therefore, for the variation implemented by a symmetry transformation $\delta_{*} \phi$, equation (1) can be rewritten under the following form

$$
\frac{\delta L}{\delta \phi} \delta_{*} \phi=-\partial_{\mu}\left(\varrho_{*}^{\mu}-\tau^{\mu}\right)
$$

where $\varrho_{*}^{\mu}=\varrho^{\mu}\left(\phi, \delta_{*} \phi\right)$. A Noether current then arises

$$
E^{\mu} \equiv E^{\mu}\left(\phi, \delta_{*} \phi\right)=\varrho_{*}^{\mu}-\tau^{\mu}
$$

which is conserved on shell. One writes

$$
\partial_{\mu} E^{\mu}=0 \quad \bmod \quad \frac{\delta L}{\delta \phi}
$$

\footnotetext{
${ }^{3}$ Here the usual Einstein summation convention over repeated indices is adopted.
} 
or, equivalently,

$$
\partial_{\mu} E^{\mu} \approx 0
$$

and calls it a weak conservation law. It follows then from the Poincaré lemma that (at least locally) there exists on shell a skew-symmetric quantity $U^{\mu \nu}=-U^{\nu \mu}$, called a superpotential, such that

$$
E^{\mu} \approx \partial_{\nu} U^{\mu \nu}
$$

i.e. $E^{\mu}$ differs from the divergence $\partial_{\nu} U^{\mu \nu}$ by a quantity which vanishes on shell. Notice that the superpotential so defined depends explicitly on field equations. However, if a formula on $U^{\mu \nu}$ can be "analytically" prolonged to all field configurations then a quantity $e^{\mu}=\partial_{\nu} U^{\mu \nu}$ defined for all field configurations is automatically conserved also "off shell", i.e.

$$
\partial_{\mu} e^{\mu} \equiv 0
$$

independently of the dynamics. In this case we call this a strong conservation law.

\section{Second Theorem of Noether and Superpoten- tials}

In the present paper we shall be interested in so-called local symmetries (and second Noether's theorem). This corresponds to the situation when the variation $\delta_{*} \phi$ depends on some (finite) number of arbitrary functions $\theta=\left\{\theta^{i}(x)\right\} i=1, \ldots, N$ over spacetime $M$ together with their derivatives up to some (finite) order, say $s$. In other words one has

$$
\delta_{*} \phi \equiv \delta_{\theta} \phi=\varphi_{i} \theta^{i}+\varphi_{i}^{\rho} \partial_{\rho} \theta^{i}+\ldots+\varphi_{i}^{\rho_{1} \ldots \rho_{s}} \partial_{\rho_{1}} \ldots \partial_{\rho_{s}} \theta^{i}
$$

The order $s$ of the highest derivative involved in this field transformation law is called the geometric order of the transformation. If the Lagrangian is of order $k$, i.e. it involves up to $k$ derivatives of the fields, the integer $r=s+k$ is then called the total order of the theory [12].

Supposing now that the total order of the theory is at most 3 , then both sides of equation (3) become differential operators acting on $\theta$, where

$$
E^{\mu} \equiv E^{\mu}(\theta)=t_{i}^{\mu} \theta^{i}+t_{i}^{\mu \rho} \partial_{\rho} \theta^{i}+t_{i}^{\mu \rho \sigma} \partial_{\rho} \partial_{\sigma} \theta^{i}
$$

\footnotetext{
${ }^{4}$ This is always the case for so-called local symmetries (see below).

${ }^{5}$ We slightly extend here the formalism developed in 13], where proofs of our statements can also be found.
} 


$$
\frac{\delta L}{\delta \phi} \delta_{\theta} \phi \equiv W(\theta)=w_{i} \theta^{i}+w_{i}^{\rho} \partial_{\rho} \theta^{i}+w_{i}^{\rho \sigma} \partial_{\rho} \partial_{\sigma} \theta^{i}
$$

Notice that in (6) and (7), in order to preserve the uniqueness of the decomposition, one should assume the following symmetry conditions for the highest coefficients .

$$
t_{i}^{\mu[\rho \sigma]}=0, \quad w_{i}^{[\rho \sigma]}=0
$$

Replacing now (6) and (7) into equation (3) and calculating the divergence $\partial_{\mu} E^{\mu}$ explicitly, one gets an identity which holds true for arbitrary functions $\theta^{i}$. Therefore the total coefficients resulting in front of $\theta^{i}, \partial_{\alpha} \theta^{i}$, etc..., should vanish independently. This produces, in fact, a differential indentity involving the Euler-Lagrange expression, so-called generalized Bianchi identity [13, which in this case reads as follows

$$
w_{i}-\partial_{\nu} w_{i}^{\nu}+\partial_{\nu} \partial_{\rho} w_{i}^{\nu \rho}=0
$$

The coefficients of $W$ as given by (7) vanish on shell because of the EulerLagrange equations, so that $\partial_{\mu} E^{\mu} \approx 0$ holds. It appears, in this case, that the Noether current admits a representation of the form

$$
E^{\mu}=\tilde{E}^{\mu}+\partial_{\rho} U^{\mu \rho} \equiv \tilde{E}^{\mu}+e^{\mu}
$$

where $\tilde{E}^{\mu}$ is the "reduced" Noether current, which vanishes on shell (see 13]). The reduced current $\tilde{E}$ is unique, while the superpotential is not, since (9) remains unchanged if $U^{\mu \nu}$ is redefined by the addition of the divergence of any skew-symmetric quantity $Y^{\mu \nu \rho}=Y^{[\nu \mu \rho]}$. It can be found that equation (9) has an explicit solution given by

$$
\begin{gathered}
\tilde{E}^{\mu}=\left(w_{i}^{\mu}-\partial_{\rho} w_{i}^{\rho \mu}\right) \theta^{i}+w_{i}^{\mu \rho} \partial_{\rho} \theta^{i} \\
U^{\mu \rho}=\left(t_{i}^{[\mu \rho]}+\partial_{\nu} \tilde{t}_{i}^{\nu[\mu \rho]}\right) \theta^{i}+\tilde{t}_{i}^{\mu \rho \nu} \partial_{\nu} \theta^{i}
\end{gathered}
$$

where $\tilde{t}_{i}^{\mu \rho \nu}=(4 / 3) t_{i}^{[\mu \rho] \nu}$. This gives explicit formulae for a superpotential in the case of theories of total order at most 3 . It should be noticed that the "lowest" coefficients $t_{i}^{\mu}$ do not enter this explicit expression (11); therefore, this suggests that superpotentials are, in a suitable sense, "algebraically simpler" then the corresponding Noether currents.

We should also remark that formula (11) seems to provide a "canonical" expression for the superpotential; but, in general, this is not true. In fact,

\footnotetext{
${ }^{6}$ We shall use the notation $A^{[\mu \nu]}=\frac{1}{2}\left(A^{\mu \nu}-A^{\nu \mu}\right)$ for the skew-symmetrisation and $A^{(\mu \nu)}=\frac{1}{2}\left(A^{\mu \nu}+A^{\nu \mu}\right)$ for the symmetrisation.
} 
equation (11) explicitly depends on the local coordinate system we have chosen as well as the transformation properties of the coefficients appearing in (6). Nevertheless, as we shall see below, such a canonical expression will be possible under some additional assumption.

As a general class of examples including theories of gravity (which in fact we shall need in the sequel) let us assume that the field $\phi$ is a field of geometric objects over $M$ [12], i.e. we assume that under coordinate changes in $M$ the field $\phi$ transforms properly through laws which depend only on a finite number of the partial derivatives of the coordinate change itself 7 . We assume also that the Lagrangian is natural (or, equivalently, reparametrization invariant), in the sense that the lift of all diffeomorphisms of $M$ transform $L$ as a scalar density of weight 1 . This means that, at the infinitesimal level, one has

$$
\delta_{*} \phi=\mathcal{L}_{\xi} \phi
$$

and

$$
\delta_{*} L=\mathcal{L}_{\xi} L=\partial_{\alpha}\left(\xi^{\alpha} L\right)
$$

where $\xi=\xi^{\alpha} \partial_{\alpha}$ is an arbitrary vectorfield on $M$ (i.e., an infinitesimal diffeomorphism) and $\mathcal{L}_{\xi}$ denotes the Lie derivative along $\xi$.

As a consequence, equation (3) can be written as follows:

$$
\partial_{\mu} E^{\mu} \equiv \partial_{\mu}\left[\varrho^{\mu}\left(\phi, \delta_{\xi} \phi\right)-\xi^{\mu} L\right]=-\frac{\delta L}{\delta \phi} \mathcal{L}_{\xi} \phi
$$

where $E^{\mu} \equiv E^{\mu}(\xi)$ is the appropriate Noether current evaluated along the infinitesimal symmetry given by $\xi$, called now energy-momentum flow. It is known that the Lie derivative of any geometric object can be expressed by means of covariant derivatives with respect to an arbitrary symmetric linear connection on $M$. In particular, we may expand the Lie derivative $\mathcal{L}_{\xi} \phi$ as a linear combination of $\xi$ and its covariant derivatives, i.e. we write

$$
\mathcal{L}_{\xi} \phi=\Phi_{\alpha} \xi^{\alpha}+\Phi_{\alpha}^{\rho} \nabla_{\rho} \xi^{\alpha}+\ldots
$$

Suppose again that the total order of the theory is at most 3. This implies global and covariant decompositions

$$
E^{\mu} \equiv E^{\mu}(\xi)=T_{\alpha}^{\mu} \xi^{\alpha}+T_{\alpha}^{\mu \rho} \nabla_{\rho} \xi^{\alpha}+T_{\alpha}^{\mu \rho \sigma} \nabla_{\rho} \nabla_{\sigma} \xi^{\alpha}
$$

\footnotetext{
${ }^{7}$ Tensors or linear connections are geometric objects, while arbitrary gauge fields are not.

${ }^{8}$ This includes all standard metric theories of gravity $(k=2, s=1)$ as well as socalled first-order formalism (or Palatini), with $k=1, s=2$ (Yang-Mills theories have $k=1, s=1$ ).
} 


$$
\frac{\delta L}{\delta \phi} \delta_{\xi} \phi \equiv W(\xi)=W_{\alpha} \xi^{\alpha}+W_{\alpha}^{\rho} \nabla_{\rho} \xi^{\alpha}+W_{\alpha}^{\rho \sigma} \nabla_{\rho} \nabla_{\sigma} \xi^{\alpha}
$$

According to the general techniques developed in 13. we get thence covariant versions of equations (9), (10) and (11), i.e.

$$
W_{\mu}-\nabla_{\nu} W_{\mu}^{\nu}+\nabla_{\nu} \nabla_{\rho} W_{\mu}^{\nu \rho}=0
$$

as a generalized Bianchi identity;

$$
\tilde{E}^{\mu}=\left(W_{\alpha}^{\mu}-\nabla_{\rho} W_{\alpha}^{\rho \mu}\right) \xi^{\alpha}+W_{\alpha}^{\mu \rho} \nabla_{\rho} \xi^{\alpha}
$$

as a reduced energy flow; and finally

$$
U^{\mu \rho}=\left(T_{\alpha}^{[\mu \rho]}+\nabla_{\nu} \tilde{T}_{\alpha}^{\nu[\mu \rho]}\right) \xi^{\alpha}+\tilde{T}_{\alpha}^{\mu \rho \nu} \nabla_{\nu} \xi^{\alpha}
$$

where $\tilde{T}_{\alpha}^{\mu \rho \nu}=(4 / 3) T_{\alpha}^{[\mu \rho] \nu}$ (recall that $\left.T_{\alpha}^{\mu[\rho \nu]}=0\right)$. Equation (19) gives the required formula for a canonical superpotential in the case of natural theories of total order at most 3 (see [13]).

As an example one can consider the superpotential obtained from the Einstein-Hilbert (purely metric) gravitational Lagrangian (here notation is standard)

$$
L_{E H}\left(g, \partial g, \partial^{2} g\right)=|\operatorname{det} g|^{\frac{1}{2}} g^{\mu \nu} R_{\mu \nu}
$$

which is given by

$$
U_{E H}^{\mu \nu}(\xi)=|\operatorname{detg}|^{\frac{1}{2}}\left(\nabla^{\mu} \xi^{\nu}-\nabla^{\nu} \xi^{\mu}\right)
$$

and it is known as the Komar superpotential ? It has been recently shown that the Komar expression is "universal" in the following sense: for a large class of non-linear gravitational Lagrangians (in the first-order "Palatini" formalism), the superpotential does not depend on the Lagrangian and is in fact equal to the Komar superpotential 10$]$ [16, 17].

In some mathematical literature currents admitting superpotentials are called trivial since they lead to strong conservation laws, which hold true irrespectively of the form of the equations of motion [18]. Such currents are also somehow "trivial" from a co-cohomological viewpoint (in the sense the they define trivial co-homology classes in de Rahm cohomology, being on

\footnotetext{
${ }^{9}$ We refere the reader to e.g. 时, 9] for more exhausted discussion of the Komar superpotential.

${ }^{10}$ It has been also found that in these cases the universality property holds for the Einstein equations as well.
} 
shell exact forms). Nevertheless, we remark that they produce physically relevant quantities, like e.g. charges, masses and so on.

The advantage of using superpotentials is twofold. First, as we have mentioned before, they are in general (algebraically) simpler expressions then the corresponding Noether currents. Moreover, in order to calculate the flux of any conserved quantity they allow, via Stokes' theorem, to reduce calculations to the following:

$$
\int_{\Sigma} E^{\mu} d \Sigma_{\mu}=\oint_{\partial \Sigma} U^{\mu \nu} d \Sigma_{\mu \nu}
$$

Here $\Sigma$ is an hypersurface in $M$ of codimension 1 and $\partial \Sigma$ denotes its boundary (which has codimension 2). In the case of $d=4$ spacetime this simply means that the flux of a conserved quantity through a 3-dimensional portion of spacetime can be calculated as the integral of the superpotential along the 2-dimensional boundary $\partial \Sigma$, which is a surface.

\section{Chern-Simons Gauge Theory}

To start with, we discuss another physically interesting example, i.e. the Chern-Simons theory considered as a gauge theory over $M=\mathbb{R}^{3}$ (see also [2, 9, 10]). The Chern-Simons three-form written in terms of a connection and its curvature reads as follows

$$
L_{C S}^{(3)}=\operatorname{tr}\left(\Omega \wedge \omega-\frac{1}{3} \omega \wedge \omega \wedge \omega\right)
$$

where $\omega=\mathbf{A}_{\mu} d x^{\mu}$ is a connection one-form, $\mathbf{A}_{\mu}$ is a matrix-valued $\boxplus$ gauge potential, $\Omega=d \omega+\omega \wedge \omega=\frac{1}{2} \mathbf{F}_{\mu \nu} d x^{\mu} \wedge d x^{\nu}$ is its curvature two-form, $\mathbf{F}_{\mu \nu}=\partial_{\mu} \mathbf{A}_{\nu}-\partial_{\nu} \mathbf{A}_{\mu}+\left[\mathbf{A}_{\mu}, \mathbf{A}_{\nu}\right]$ is the gauge field strength and $\operatorname{tr}$ denotes the matrix trace.

The corresponding Lagrangian density $L_{C S}^{(3)}=L_{C S} d x^{1} \wedge d x^{2} \wedge d x^{3}$ written in terms of more physically relevant quantities is

$$
L_{C S}=\frac{1}{2} \varepsilon^{\mu \nu \rho} \operatorname{tr}\left(\mathbf{F}_{\mu \nu} \mathbf{A}_{\rho}-\frac{2}{3} \mathbf{A}_{\mu} \mathbf{A}_{\nu} \mathbf{A}_{\rho}\right)
$$

where $\varepsilon^{\mu \nu \rho}$ is the totally skew-symmetric Levi-Civita symbol in $d=3$.

\footnotetext{
${ }^{11}$ In fact, $\mathbf{A}_{\mu}$ belongs to the Lie algebra of some gauge group $G$, which for simplicity we assume here to be represented in terms of (complex) matrices.
} 
In this case the first variation formula (1) reads as

$$
\delta L_{C S}=\varepsilon^{\mu \nu \rho} \operatorname{tr}\left(\mathbf{F}_{\mu \nu} \delta \mathbf{A}_{\rho}\right)-\partial_{\mu}\left[\varepsilon^{\mu \nu \rho} \operatorname{tr}\left(\mathbf{A}_{\nu} \delta \mathbf{A}_{\rho}\right)\right]
$$

and this gives $\mathbf{F}_{\mu \nu}=0$ as the equation of motion, which means $\Omega=0$, i.e. that only flat connections are solutions of the equations of motion.

As a symmetry one can consider any infinitesimal gauge transformation

$$
\delta_{\chi} \mathbf{A}_{\mu}=D_{\mu} \chi
$$

where $\chi$ is an arbitrary (matrix-valued) function and $D_{\mu}=\partial_{\mu}+\left[\mathbf{A}_{\mu}, \cdot\right]$ denotes the covariant derivative with respect to the connection $\omega$. It is known from transformation properties of the Lagrangian $L_{C S}$ (see e.g. [2, 6]) that the following holds:

$$
\delta_{\chi} L_{C S}=\partial_{\mu}\left[\varepsilon^{\mu \nu \rho} \operatorname{tr}\left(\mathbf{A}_{\nu} \partial_{\rho} \chi\right)\right]
$$

Replacing now (22), (23) and (24) into equation (3) we get

$$
\varepsilon^{\mu \nu \rho} \operatorname{tr}\left(\mathbf{F}_{\mu \nu} D_{\rho} \chi\right)=\partial_{\mu} E_{C S}^{\mu}(\chi)
$$

where

$$
\begin{gathered}
E_{C S}^{\mu}(\chi)=2 \varepsilon^{\mu \nu \rho} \operatorname{tr}\left(\mathbf{A}_{\nu} \mathbf{A}_{\rho} \chi\right)+2 \varepsilon^{\mu \nu \rho} \operatorname{tr}\left(\mathbf{A}_{\nu} \partial_{\rho} \chi\right)= \\
-2 \varepsilon^{\mu \nu \rho} \operatorname{tr}\left(\mathbf{A}_{\nu} \mathbf{A}_{\rho} \chi\right)+2 \varepsilon^{\mu \nu \rho} \operatorname{tr}\left(\mathbf{A}_{\nu} D_{\rho} \chi\right)
\end{gathered}
$$

is the expression for the Noether current stemming from the gauge invariance of $I_{C S}$. Therefore, indentifying the corresponding coefficients in (26) with those in (7), we obtain with the help of (11) the following superpotential (see also [9]):

$$
U_{C S}^{\mu \rho}(\chi)=2 \varepsilon^{\mu \nu \rho} \operatorname{tr}\left(\mathbf{A}_{\nu} \chi\right)
$$

The left hand side of (25), after expanding it as a differential operator acting on $\chi$, gives rise (see also equation (8)) to the generalized Bianchi identity

$$
\varepsilon^{\mu \nu \rho} D_{\rho} \mathbf{F}_{\mu \nu}=0
$$

which, in dimension $d=3$, is known to be equivalent to the standard Bianchi identity for the gauge field $\mathbf{F}_{\mu \nu}$, i.e.

$$
D_{\rho} \mathbf{F}_{\mu \nu}+D_{\mu} \mathbf{F}_{\nu \rho}+D_{\nu} \mathbf{F}_{\rho \mu}=0
$$


A similar analysis can be performed for the diffeomorphism invariance of $L_{C S}$; in this case one has [2:

$$
\delta_{\xi} \mathbf{A}_{\mu} \equiv \mathcal{L}_{\xi} \mathbf{A}_{\mu}=\xi^{\alpha} \partial_{\alpha} \mathbf{A}_{\mu}+\mathbf{A}_{\alpha} \partial_{\mu} \xi^{\alpha}
$$

and

$$
\delta_{\xi} L_{C S} \equiv \mathcal{L}_{\xi} L_{C S}=\partial_{\alpha}\left(\xi^{\alpha} L_{C S}\right)
$$

Now we have:

$$
E^{\mu}(\xi)=\xi^{\mu} L_{C S}+\varepsilon^{\mu \nu \rho} \operatorname{tr}\left(\mathbf{A}_{\nu} \partial_{\alpha} \mathbf{A}_{\rho}\right) \xi^{\alpha}+\varepsilon^{\mu \nu \rho} \operatorname{tr}\left(\mathbf{A}_{\nu} \mathbf{A}_{\alpha}\right) \partial_{\rho} \xi^{\alpha}
$$

from which it follows via (11)

$$
U_{C S}^{\mu \rho}(\xi)=\varepsilon^{\mu \nu \rho} \operatorname{tr}\left(\mathbf{A}_{\nu} \mathbf{A}_{\alpha}\right) \xi^{\alpha}
$$

since only the last term in (31) contributes into (32).

The generalized Bianchi identity takes thence the form

$$
\varepsilon^{\mu \nu \rho} \operatorname{tr}\left(D_{\rho} \mathbf{F}_{\mu \nu} \mathbf{A}_{\alpha}-\mathbf{F}_{\mu \nu} \mathbf{F}_{\alpha \rho}\right)=0
$$

in which the first term vanishes owing to (28), so that (33) reduces in fact to the funny algebraic identity

$$
\varepsilon^{\mu \nu \rho} \operatorname{tr}\left(\mathbf{F}_{\mu \nu} \mathbf{F}_{\alpha \rho}\right)=0
$$

which holds true for any gauge field in dimension three!

For a comparison, we recall the reader that the gauge and diffeomorphism invariance of Yang-Mills Lagrangians in four dimensions lead instead to

$$
\begin{gathered}
U_{Y M}^{\mu \rho}(\chi)=\operatorname{tr}\left(\mathbf{F}^{\mu \rho} \chi\right) \\
U_{Y M}^{\mu \rho}(\xi)=\operatorname{tr}\left(\mathbf{F}^{\mu \rho} \mathbf{A}_{\alpha}\right) \xi^{\alpha}
\end{gathered}
$$

Some autors [20, 21] prefer to deal with so called improved diffeomorphisms

$$
\hat{\delta}_{\xi} \mathbf{A}_{\mu} \equiv \xi^{\alpha} \mathbf{F}_{\alpha \mu}=\delta_{\xi} \mathbf{A}_{\mu}-D_{\mu}\left(\xi^{\alpha} \mathbf{A}_{\alpha}\right)
$$

which differ from the Lie derivative (29) only by a gauge transformation $\hat{\chi}=\xi^{\alpha} \mathbf{A}_{\alpha}$. Direct calculations show that

$$
\hat{U}_{C S}^{\mu \rho}(\xi)=U_{C S}^{\mu \rho}(\xi)-U_{C S}^{\mu \rho}(\hat{\chi})=\varepsilon^{\mu \rho \nu} \operatorname{tr}\left(\mathbf{A}_{\nu} \mathbf{A}_{\alpha}\right) \xi^{\alpha}
$$

i.e., it differs from (32) by a sign. Similarly, one finds from (35-37)

$$
\hat{U}_{Y M}^{\mu \rho}(\xi)=0
$$

It means that there are no, in the Yang-Mills theory, non-trivial charges corresponding to these transformations.

\footnotetext{
${ }^{12}$ Under diffeomorphisms the gauge potential $\mathbf{A}_{\mu}$ behaves as a one-form and $L_{C S}$ as a scalar density of weight one.
} 


\section{Chern-Simons Gravity}

We now proceed to discuss a less studied case of Chern-Simons gravity considered as a purely metric theory over $\mathbb{R}^{3}$. Therefore, the only dynamical variable is a Riemannian (or pseudo-Riemannian) metric $\mathbf{g}=g_{\mu \nu}$. The Lagrangian density is

$$
\begin{gathered}
L_{C S G}=\frac{1}{2} \varepsilon^{\mu \nu \rho} \operatorname{tr}\left(\mathbf{R}_{\mu \nu} \boldsymbol{\Gamma}_{\rho}-\frac{2}{3} \boldsymbol{\Gamma}_{\mu} \boldsymbol{\Gamma}_{\nu} \boldsymbol{\Gamma}_{\rho}\right) \\
=\frac{1}{2} \varepsilon^{\mu \nu \rho}\left(R_{\beta \mu \nu}^{\alpha} \Gamma_{\alpha \rho}^{\beta}-\frac{2}{3} \Gamma_{\beta \mu}^{\alpha} \Gamma_{\sigma \nu}^{\beta} \Gamma_{\alpha \rho}^{\sigma}\right)
\end{gathered}
$$

where, using the notation introduced in the previous section, we have now $\omega=\boldsymbol{\Gamma}_{\mu} d x^{\mu}, \boldsymbol{\Gamma}_{\mu}$ is the matrix-valued $[3$ one-form of Riemannian connection, $\Omega=\frac{1}{2} \mathbf{R}_{\mu \nu} d x^{\mu} \wedge d x^{\nu}$ is the Riemann curvature two-form and $\mathbf{R}_{\mu \nu}=\partial_{\mu} \boldsymbol{\Gamma}_{\nu}-$ $\partial_{\nu} \boldsymbol{\Gamma}_{\mu}+\left[\boldsymbol{\Gamma}_{\mu}, \boldsymbol{\Gamma}_{\nu}\right]$ is the Riemann curvature tensor written in matrix notation. For the sake of completeness we also recall two basic formulae of Riemannian geometry

$$
\begin{gathered}
\Gamma_{\beta \mu}^{\alpha}=\frac{1}{2} g^{\alpha \sigma}\left(\partial_{\beta} g_{\mu \sigma}+\partial_{\mu} g_{\sigma \beta}-\partial_{\sigma} g_{\beta \mu}\right) \\
R_{\beta \mu \nu}^{\alpha}=\partial_{\mu} \Gamma_{\beta \nu}^{\alpha}-\partial_{\nu} \Gamma_{\beta \mu}^{\alpha}+\Gamma_{\sigma \mu}^{\alpha} \Gamma_{\beta \nu}^{\sigma}-\Gamma_{\sigma \nu}^{\alpha} \Gamma_{\beta \mu}^{\sigma}
\end{gathered}
$$

as well as the following indentity

$$
R_{\beta \mu \nu}^{\alpha}=\delta_{\mu}^{\alpha} R_{\beta \nu}-\delta_{\nu}^{\alpha} R_{\beta \mu}+g_{\beta \nu} R_{\mu}^{\alpha}-g_{\beta \mu} R_{\nu}^{\alpha}+\frac{1}{2} R\left(g_{\beta \mu} \delta_{\nu}^{\alpha}-g_{\beta \nu} \delta_{\mu}^{\alpha}\right)
$$

which holds true only in dimension $d=3$ (see e.g. 19]) [4. Here, $R_{\mu \nu} \equiv R_{\mu \alpha \nu}^{\alpha}$ denotes the Ricci tensor and $R=R_{\alpha}^{\alpha}$ is the Ricci scalar 10 of $\mathbf{g}$.

Notice that $L_{C S G}$ depends on the metric $\mathbf{g}$ only through the Riemannian connection (41). The relation (22) remains still valid

$$
\delta L_{C S G}=\varepsilon^{\mu \nu \rho} \operatorname{tr}\left(\mathbf{R}_{\mu \nu} \delta \boldsymbol{\Gamma}_{\rho}\right)-\partial_{\mu}\left[\varepsilon^{\mu \nu \rho} \operatorname{tr}\left(\boldsymbol{\Gamma}_{\nu} \delta \boldsymbol{\Gamma}_{\rho}\right)\right]
$$

but it does not correspond now to the first variation formula (1), since $\boldsymbol{\Gamma}_{\rho}$ is no longer a dynamical variable in our theory. Accordingly, one has to

\footnotetext{
${ }^{13}$ Here $\boldsymbol{\Gamma}_{\mu} \equiv \Gamma_{\beta \mu}^{\alpha}$ and $\mathbf{R}_{\mu \nu} \equiv R_{\beta \mu \nu}^{\alpha}$ are represented as $3 \times 3$ matrices and the upper index is the row index.

${ }^{14}$ This means, in fact, that in three dimensions the two notions of Ricci flatness and flatness coincide: i.e., that gravity is trivial in dimension $d=3$. This is why a coupling with Chern-Simons term enriches the theory.

${ }^{15}$ We shall be frequently using $\mathbf{g}$ for raising and lowering indices.
} 
replace $\delta \boldsymbol{\Gamma}_{\rho}$ in the first term by means of the well known "Palatini formula"

$$
\delta \Gamma_{\beta \rho}^{\alpha}=\frac{1}{2} g^{\alpha \sigma}\left(\nabla_{\beta} \delta g_{\rho \sigma}+\nabla_{\rho} \delta g_{\sigma \beta}-\nabla_{\sigma} \delta g_{\beta \rho}\right)
$$

Making use of equation (43) together with the contracted Bianchi identity $\nabla_{\alpha} R_{\mu}^{\alpha}=\frac{1}{2} \nabla_{\mu} R$ one finds

$$
\varepsilon^{\mu \nu \rho} R_{\alpha \mu \nu}^{\beta} \delta \Gamma_{\beta \rho}^{\alpha}=-2 \varepsilon^{\mu \nu \rho} \nabla_{\mu} R_{\nu}^{\alpha} \delta g_{\alpha \rho}+\partial_{\mu}\left(2 \varepsilon^{\mu \nu \rho} R_{\nu}^{\alpha} \delta g_{\alpha \rho}\right)
$$

With the help of the tricky identity which holds true for any quantity $S_{\mu \nu}^{\sigma}$

$$
2 \varepsilon^{\mu \nu[\rho} S_{\mu \nu}^{\sigma]} \equiv \varepsilon^{\rho \sigma \beta}\left(S_{\alpha \beta}^{\alpha}-S_{\beta \alpha}^{\alpha}\right)
$$

the symmetric part of the first term of the right hand side in (45) can be converted into the following form

$$
-2 \varepsilon^{\mu \nu \rho} \nabla_{\mu}\left(R_{\nu}^{\alpha}-\frac{1}{4} \delta_{\nu}^{\alpha} R\right) \delta g_{\alpha \rho}
$$

Therefore, the first variation formula reads now as

$$
\delta L_{C S G}=-2 C^{\alpha \rho} \delta g_{\alpha \rho}+\partial_{\mu}\left[\varepsilon^{\mu \nu \rho}\left(2 R_{\nu}^{\alpha} \delta g_{\alpha \rho}-\operatorname{tr}\left(\boldsymbol{\Gamma}_{\nu} \delta \boldsymbol{\Gamma}_{\rho}\right)\right)\right]
$$

where

$$
C^{\alpha \rho}=\varepsilon^{\mu \nu \rho} \nabla_{\mu}\left(R_{\nu}^{\alpha}-\frac{1}{4} \delta_{\nu}^{\alpha} R\right)
$$

is the so-called Cotton tensor 7 , the vanishing of which gives, in the case of $L_{C S G}$, the Euler-Lagrange equations of motion. It is also known (see e.g. [2, 19]) that the Cotton tensor is symmetric, traceless $\left(C_{\alpha}^{\alpha}=0\right)$, divergencefree $\left(\nabla_{\alpha} C_{\beta}^{\alpha}=0\right)$ and it vanishes if and only if the metric $\mathbf{g}$ is conformally flat (三 conformal to a flat one).

As a symmetry transformation, consider then a 1-parameter group of diffeomorphisms generated by the vectorfield $\xi=\xi^{\alpha} \partial_{\alpha}$. In this case one can use the well known expressions

$$
\delta_{\xi} \mathbf{g} \equiv \mathcal{L}_{\xi} g_{\alpha \rho}=\nabla_{\alpha} \xi_{\rho}+\nabla_{\rho} \xi_{\alpha}
$$

and

$$
\delta_{\xi} \boldsymbol{\Gamma}_{\rho} \equiv \mathcal{L}_{\xi} \Gamma_{\alpha \rho}^{\beta}=\xi^{\sigma} R_{\alpha \sigma \rho}^{\beta}+\nabla_{\alpha} \nabla_{\rho} \xi^{\beta}
$$

\footnotetext{
${ }^{16}$ In fact, $C^{\alpha \rho}$ is a tensor denity of weight 1.
} 
In a Riemannian case we are dealing with, the last formula is, of course, equivalent to the Palatini one, if one replaces $\delta g$ in (44) by (49).

Thus, our task is now to calculate $\delta_{\xi} L_{C S G}$ directly from its transformation properties. Notice that the transformation rule for the linear connection

$$
\begin{aligned}
& \bar{\Gamma}_{\nu \mu}^{\lambda}=\frac{\partial \bar{x}^{\lambda}}{\partial x^{\rho}} \Gamma_{\alpha \beta}^{\rho} \frac{\partial x^{\alpha}}{\partial \bar{x}^{\nu}} \frac{\partial x^{\beta}}{\partial \bar{x}^{\mu}}+\frac{\partial \bar{x}^{\lambda}}{\partial x^{\rho}} \frac{\partial^{2} x^{\rho}}{\partial \bar{x}^{\nu} \partial \bar{x}^{\mu}} \\
& =\left(\frac{\partial \bar{x}^{\lambda}}{\partial x^{\rho}} \Gamma_{\alpha \beta}^{\rho} \frac{\partial x^{\alpha}}{\partial \bar{x}^{\nu}}+\frac{\partial \bar{x}^{\lambda}}{\partial x^{\rho}} \frac{\partial}{\partial x^{\beta}}\left[\frac{\partial x^{\rho}}{\partial \bar{x}^{\nu}}\right]\right) \frac{\partial x^{\beta}}{\partial \bar{x}^{\mu}}
\end{aligned}
$$

is the composition of a vector- and of a pure gauge transformation. In other words one has

$$
\overline{\boldsymbol{\Gamma}}_{\mu}=\left(\mathbf{J} \boldsymbol{\Gamma}_{\beta} \mathbf{J}^{-1}+\mathbf{J} \partial_{\beta} \mathbf{J}^{-1}\right) \frac{\partial x^{\beta}}{\partial \bar{x}^{\mu}}
$$

where $\mathbf{J}=\frac{\partial \bar{x}^{\lambda}}{\partial x^{\rho}}$ is the Jacobian matrix. Therefore, at the infinitesimal level $\left(\bar{x}^{\alpha}=x^{\alpha}-\xi^{\alpha}\right), \delta_{\xi} L_{C S G}$ is the sum of two terms $\square$ of the type (24) and (13). More precisely we have obtained

$$
\delta_{\xi} L_{C S G}=\partial_{\mu}\left[\xi^{\mu} L_{C S G}+\varepsilon^{\mu \nu \rho} \operatorname{tr}\left(\boldsymbol{\Gamma}_{\nu} \partial_{\rho} \bar{\chi}\right)\right]
$$

where $\bar{\chi}=\frac{\partial \xi^{\beta}}{\partial x^{\alpha}}$ is an infinitesimal version of the inverse Jacobian matrix. We see that the Lagrangian $L_{C S G}$ is not natural, but it is in some sense quasi-natural, i.e. it differs from a natural one by a total derivative.

In a similar way one deduces from (51) that the following holds

$$
\delta_{\xi} \boldsymbol{\Gamma}_{\rho}=\nabla_{\rho} \bar{\chi}+\xi^{\alpha} \partial_{\alpha} \boldsymbol{\Gamma}_{\rho}+\boldsymbol{\Gamma}_{\alpha} \partial_{\rho} \xi^{\alpha}
$$

as the sum of (23) and (29). Here $\nabla_{\rho} \bar{\chi}$ denotes the "formal" covariant differential of $\bar{\chi}$, formally treated as a tensor of rank $(1,1)$, i.e. we set $\nabla_{\rho} \bar{\chi}=\partial_{\rho} \bar{\chi}+\left[\boldsymbol{\Gamma}_{\rho}, \bar{\chi}\right]$. Of course, it can be directly checked that equations (50) and (53) are equivalent.

Inserting now all necessary expressions in equation (3) we obtain the variation formula under the form

$$
2 C^{\alpha \rho} \mathcal{L}_{\xi} g_{\alpha \rho}=-\partial_{\mu} E_{C S G}^{\mu}(\xi)
$$

where

$$
E_{C S G}^{\mu}(\xi)=\xi^{\mu} L_{C S G}+\varepsilon^{\mu \nu \rho}\left\{\operatorname{tr}\left(\boldsymbol{\Gamma}_{\nu} \partial_{\alpha} \boldsymbol{\Gamma}_{\rho}\right) \xi^{\alpha}-2 R_{\nu}^{\tau}\left(\delta_{\tau}^{\sigma} g_{\rho \alpha}+\delta_{\rho}^{\sigma} g_{\tau \alpha}\right) \nabla_{\sigma} \xi^{\alpha}+\right.
$$

\footnotetext{
${ }^{17}$ In fact, we have to substitute $\chi \mapsto \frac{\partial \xi^{\beta}}{\partial x^{\alpha}}$ in equation (24).
} 


$$
\left.+\operatorname{tr}\left(\boldsymbol{\Gamma}_{\nu}\left[\boldsymbol{\Gamma}_{\rho}, \bar{\chi}\right]\right)+\operatorname{tr}\left(\boldsymbol{\Gamma}_{\nu} \boldsymbol{\Gamma}_{\alpha}\right) \partial_{\rho} \xi^{\alpha}+2 \operatorname{tr}\left(\boldsymbol{\Gamma}_{\nu} \partial_{\rho} \bar{\chi}\right)\right\}
$$

is the energy-momentum complex for the Chern-Simons gravitational Lagrangian (40). We are now in position to obtain the corresponding superpotential $U_{C S G}^{\mu \rho}$. Since superpotentials are additive quantities one can calculate the contributions from all terms of (55) separately. Moreover, the first two terms are of the lowest order and do not contribute to the superpotential.

To obtain the contribution from the third term of (55) one will use the covariant formula (19). First we rewrite this term under the form $T_{\alpha}^{\mu \sigma} \nabla_{\sigma} \xi^{\alpha}$, where $T_{\alpha}^{\mu \sigma}=-2 \varepsilon^{\mu \nu \rho} R_{\nu}^{\beta}\left(\delta_{\beta}^{\sigma} g_{\rho \alpha}+\delta_{\rho}^{\sigma} g_{\beta \alpha}\right)$. Then, by making use of the identity (46), $T_{\alpha}^{[\mu \sigma]}$ can be converted into the explicit skew-symmetric form $\varepsilon^{\mu \sigma \nu}\left(3 R_{\nu \alpha}-R g_{\nu \alpha}\right)$. This gives rise to:

$$
{ }^{3} U_{C S G}^{\mu \rho}=\varepsilon^{\mu \nu \rho}\left(R g_{\nu \alpha}-3 R_{\nu \alpha}\right) \xi^{\alpha}
$$

Similarly, contributions coming from the fourth and the fifth terms are easy to calculate directly by using (11) and (46):

$$
{ }^{4} U_{C S G}^{\mu \rho}+{ }^{5} U_{C S G}^{\mu \rho}=\varepsilon^{\mu \nu \rho}\left\{\left[\boldsymbol{\Gamma}_{\sigma}, \boldsymbol{\Gamma}_{\nu}\right]_{\alpha}^{\sigma}+\operatorname{tr}\left(\boldsymbol{\Gamma}_{\nu} \boldsymbol{\Gamma}_{\alpha}\right)\right\} \xi^{\alpha}
$$

where $\left[\boldsymbol{\Gamma}_{\sigma}, \boldsymbol{\Gamma}_{\nu}\right]_{\alpha}^{\sigma}=\Gamma_{\beta \sigma}^{\sigma} \Gamma_{\alpha \nu}^{\beta}-\Gamma_{\beta \nu}^{\sigma} \Gamma_{\alpha \sigma}^{\beta}$, which due to the symmetry of $\Gamma_{\alpha \sigma}^{\beta}$ reduces (57) to the form:

$$
{ }^{4} U_{C S G}^{\mu \rho}+{ }^{5} U_{C S G}^{\mu \rho}=\varepsilon^{\mu \nu \rho}\left(\Gamma_{\beta \sigma}^{\sigma} \Gamma_{\alpha \nu}^{\beta}\right) \xi^{\alpha}
$$

The sixth and last term in (55) is of the second order. We write it as $t_{\alpha}^{\mu \rho \sigma} \partial_{\rho} \partial_{\sigma} \xi^{\alpha}$, with $t_{\alpha}^{\mu \rho \sigma}=2 \varepsilon^{\mu \nu(\rho} \Gamma_{\alpha \nu}^{\sigma)}$. One needs the shorthand $\tilde{t}_{\alpha}^{\mu \rho \sigma}=$ $4 / 3 t_{\alpha}^{[\mu \rho] \sigma}$ (see (11)); a direct calculation with the help of another tricky identity

$$
2 \varepsilon^{\mu \nu[\rho} A_{\nu}^{\sigma]} \equiv \varepsilon^{\rho \sigma \nu}\left(A_{\nu}^{\mu}-\delta_{\nu}^{\mu} A_{\beta}^{\beta}\right)
$$

gives then rise to $\tilde{t}_{\alpha}^{\mu \rho \sigma}=2 \varepsilon^{\mu \nu \rho} \Gamma_{\alpha \nu}^{\sigma}+(2 / 3) \varepsilon^{\mu \rho \sigma} \Gamma_{\alpha \beta}^{\beta}$. Since ${ }^{6} U_{C S G}^{\mu \rho}=\partial_{\nu} \tilde{t}_{\alpha}^{[\mu \rho]} \xi^{\alpha}+$ $\tilde{t}_{\alpha}^{\mu \rho \sigma} \partial_{\sigma} \xi^{\alpha}$, one gets:

$$
{ }^{6} U_{C S G}^{\mu \rho}=\frac{1}{3} \varepsilon^{\mu \nu \rho}\left(\partial_{\nu} \Gamma_{\alpha \beta}^{\beta}-3 \partial_{\beta} \Gamma_{\alpha \nu}^{\beta}\right) \xi^{\alpha}+\frac{2}{3} \varepsilon^{\mu \nu \rho}\left(3 \Gamma_{\alpha \nu}^{\sigma}-\delta_{\nu}^{\sigma} \Gamma_{\alpha \beta}^{\beta}\right) \partial_{\sigma} \xi^{\alpha}
$$

We can now formulate our main result: the superpotential $U_{C S G}^{\mu \rho}(\xi)$ appears as the sum:

$$
U_{C S G}^{\mu \rho}(\xi)={ }^{3} U_{C S G}^{\mu \rho}+{ }^{4} U_{C S G}^{\mu \rho}+{ }^{5} U_{C S G}^{\mu \rho}+{ }^{6} U_{C S G}^{\mu \rho}
$$

with addenda given by (56), (58) and (60).

The left hand side of (54) taking into account (49) has the form $4 C_{\beta}^{\alpha} \nabla_{\alpha} \xi^{\beta}$. This, of course, leads via (17) to a divergence-free property of the Cotton tensor as a generalized Bianchi identity. 


\section{Acknowledgments}

One of us (A.B.) gratefully acknowledges the hospitality of the Department of Mathematics of the University of Torino under the support of G.N.F.M. of Italian C.N.R. This work is sponsored by G.N.F.M., M.U.R.S.T. (40\% Proj. "Metodi Geometrici e Probabilistici in Fisica Matematica"); one of us (A. B.) acknowledges also support from KBN 2 P302 02307.

\section{References}

[1] S. S. Chern and J. Simons, Proc. Nat. Acad. Sci. USA 68(4) (1971), 791 S. S. Chern and J. Simons, Ann. Math. 99 (1974), 48

[2] S. Deser, R. Jackiw and S. Templeton, Phys. Rev. Lett. 48 (1982), 975

S. Deser, R. Jackiw and S. Templeton, Ann. of Physics 140 (1982), 372

[3] S. Deser, Chern-Simons Terms as an Example of the Relations Between Mathematics and Physics, math-ph/9805020)

[4] E. Witten, Commun. Math. Phys. 121 (1989), 351

[5] I. M. Anderson, Ann. of Math., 120 (1984) 329

[6] A. H. Chamseddine and J. Fröhlich, Commun. Math. Phys. 147 (1992), 549

[7] J. N. Goldberg, Phys. Rev. 111 (1958), 315

J. N. Goldberg, Phys. Rev. D41(2) (1990), 410

[8] J. G. Fletcher, Rev. Mod. Phys. 32 (1) (1960), 65

[9] D. Bak, D. Cangemi and R. Jackiw, Phys. Rev. D49 (1994), 5173

[10] F. W. Hehl, J. D. McCrea, E. W. Mielke and Y. Ne'eman, Phys. Rep. 258 (1995), 1

[11] B. Julia and S. Silva, Class. Quantum Grav. 15 (1998), 2173

S. Silva, On Superpotentials and Charge Algebras of Gauge Theories hep-th/9809109

[12] M. Ferraris and M. Francaviglia, Journ. Math. Phys. 26(6) (1985), 1243 
[13] M. Ferraris, M. Francaviglia and O. Robutti, in: Géométrie et Physique (Proceedings Journè̀es Relativistes de Marseille 1985); Y. ChoquetBruhat, B. Coll, R. Kerner and A. Lichnerowicz eds.; Travaux en Cours, Hermann (Paris, 1987), pp. 112-125

M. Ferraris and M. Francaviglia, Class. Quantum Grav. 9, (Supplement 1992), S79

[14] L. Fatibene, M. Ferraris and M. Francaviglia, Journ. Math. Phys. 38(8) (1997), 3953

[15] G. Sardanashvily, Class. Quantum Grav. 14 (1997), 1371

G. Giachetta, L. Mangiarotti and G. Sardanashvily, Energy-Momentum and Gauge Conservation Laws, (gr-qc/9807054)

[16] A. Borowiec, M. Ferraris, M. Francaviglia and I. Volovich, Gen. Rel. Grav. 26(7) (1994), 637

[17] A. Borowiec, M. Ferraris, M. Francaviglia and I. Volovich, Class. Quantum Grav. 15(1) (1998), 43 (gr-qc/9611067)

A. Borowiec and M. Francaviglia, Alternative Lagrangians for Einstein Metrics, Proc. Int. Sem. Math. Cosmol., Potsdam 1998, M. Rainer and H.-J. Schmidt (eds.), WSPC - to appear; (gr-qc/9806116)

[18] C. G. Torre and I. M. Anderson, Phys. Rev. Lett. 70(23) (1993), 3525

[19] J. W. York, Phys. Rev. Lett. 26(26) (1971), 1656

[20] R. Jackiw, Phys. Rev. Lett. 41(24) (1978), 1635

[21] M. Bañados L. J. Garay and M. Henneaux, Phys. Rev. D53(2) (1996), R593

M. Bañados, M. Henneaux, C. Iannuzzo and C. M. Viallet, Class. Quantum Grav. 14 (1997), 2455 